\title{
FRECUENCIA DE RESISTENCIA A LA COLISTINA EN Pseudomonas aeruginosa: PRIMER REPORTE EN EL PERÚ
}

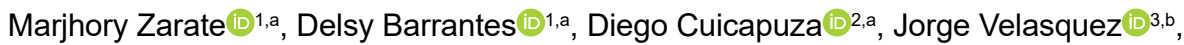 \\ Nathaly Fernández (i] ${ }^{3, b}$, Guillermo Salvatierra (iD) $2, c$, Jesus Tamariz ${ }^{1,1, d}$
${ }^{1}$ Laboratorio de Resistencia Antimicrobiana e Inmunopatología, Universidad Peruana Cayetano Heredia, Lima, Perú.
${ }^{2}$ Laboratorio de Genómica Microbiana, Facultad de Ciencias y Filosofía, Universidad Peruana Cayetano Heredia, Lima, Perú.
${ }^{3}$ Hospital Nacional Arzobispo Loayza, Lima, Perú. \\ ${ }^{a}$ Tecnólogo médico; ${ }^{b}$ médico patólogo clínico; ${ }^{\mathrm{c}}$ médico veterinario; ${ }^{\mathrm{d}}$ microbiólogo, doctor en Ciencias
}

El presente estudio forma parte de la tesis de licenciatura de Delsy Barrantes Salinas y Marjhory Zarate Estrada, «Resistencia antimicrobiana a colistina en Pseudomonas aeruginosa aisladas de tres establecimientos de salud de Lima, Perú», Facultad de Medicina Honorio Delgado; Universidad Peruana Cayetano Heredia, presentada en el 2020.

\section{RESUMEN}

El objetivo del estudio fue determinar la frecuencia de resistencia a la colistina en Pseudomonas aeruginosa provenientes de tres establecimientos de salud de Lima, criopreservados en el banco de cepas del Laboratorio de Resistencia a Antimicrobianos e Inmunopatología de la Universidad Peruana Cayetano Heredia (UPCH). El método de elución de discos de colistina en caldo fue empleado para la identificación fenotípica de la resistencia a la colistina; la detección de la expresión del gen $m c r-1$ se realizó mediante el método fenotípico de difusión de discos combinados de colistina y ácido etilendiaminotetraacético (EDTA) y la reacción en cadena de la polimerasa (PCR) para la identificación molecular del gen. De los 97 aislados estudiados, 7 (7,2\%) fueron resistentes a la colistina y ninguno fue portador del gen $m c r-1$. Este estudio constituye el primer reporte en el Perú de aislados clínicos de Pseudomonas aeruginosa resistentes a la colistina, lo que implica la necesidad de implementar metodologías apropiadas para la vigilancia epidemiológica de patógenos resistentes a la colistina.

Palabras clave: Pseudomonas aeruginosa; Farmacorresistencia Microbiana; Colistina; Perú (Fuente: DeCS BIREME).

\section{FREQUENCY OF COLISTIN RESISTANCE IN Pseudomonas aeruginosa: FIRST REPORT FROM PERU}

Citar como: Zarate M, Barrantes D, Cuicapuza D, Velasquez J, Fernández $\mathrm{N}$, Salvatierra G, et al. Frecuencia de resistencia a colistina en Pseudomonas aeruginosa: primer reporte en el Perú. Rev Peru Med Exp Salud Publica. 2021;38(2):308-12. doi: https://doi. org/10.17843/rpmesp.2021.382.6977.

Correspondencia:

Jesus Tamariz-Ortiz;

Av. Honorio Delgado 430, Urb. Ingeniería, San Martín de Porres, Lima, Perú; jesus.tamariz@upch.pe

Recibido: $29 / 12 / 2020$

Aprobado: 07/04/2021

En Línea: 26/05/2021

\begin{abstract}
This study aimed to determine the frequency of colistin resistance in Pseudomonas aeruginosa isolates obtained from three healthcare facilities in Lima and cryopreserved at the Laboratorio de Resistencia Antimicrobianos e Inmunopatología of the Universidad Peruana Cayetano Heredia (UPCH). The colistin broth disk elution method was used for the phenotypic identification of colistin resistance. We detected the expression of the mcr-1 gene by using the phenotypic diffusion method with combined colistin and ethylenediaminetetraacetic acid (EDTA) disks; and polymerase chain reaction (PCR) was used for molecular identification of the gene. Of the 97 isolates, 7 (7.2\%) were resistant to colistin; however, none carried the $m c r-1$ gene. This is the first report from Peru on clinical isolates of colistin-resistant Pseudomonas aeruginosa, which suggests the need for implementation of appropriate methodologies for the epidemiological surveillance of colistin-resistant pathogens.
\end{abstract}

Keywords: Pseudomonas aeruginosa; Antimicrobial Drug Resistance; Colistin; Peru (Source: MeSH NLM).

\section{INTRODUCCIÓN}

Pseudomona aeruginosa es un patógeno oportunista de relevancia clínica y epidemiológica, se asocia a infecciones nosocomiales que incluyen: sepsis, neumonías e infecciones del tracto urinario. Debido a los mecanismos de resistencia intrínseca, adquirida y adaptativa que caracterizan al patógeno, las alternativas terapéuticas de las infecciones que produce se limitan solo a algunos grupos de antimicrobianos. Sin embargo, la alta presión selectiva que ejercen los antimicrobianos en ambientes hospitalarios ha generado una rápida propagación y dise- 
minación mundial de clones de P. aeruginosa multidrogorresistentes (MDR) ${ }^{(1)}$.

La preocupación relacionada a esta bacteria ha sido destacada por la Organización Mundial de la Salud (OMS) y en febrero del 2017 incluyó a $P$. aeruginosa resistente a carbapenémicos en la lista de patógenos con prioridad crítica, para los que se requiere nuevos antibióticos y que ha obligado a adoptar estrategias de tratamientos más agresivas, por ejemplo, el uso de antiguos antibióticos como la colistina, pese a su elevada toxicidad ${ }^{(2)}$.

La colistina es un antibiótico cíclico polipeptídico de naturaleza lipídica catiónica descubierto en 1947. Actúa a nivel de los lipopolisacáridos (LPS) de la membrana externa de los bacilos gramnegativos (BNG), uniéndose electrostáticamente al lípido A, desplazando los cationes de $\mathrm{Mg} 2+$ y $\mathrm{Ca} 2+$ e induciendo la apertura de la membrana externa, cambios osmóticos en el periplasma y la posterior lisis bacteriana ${ }^{(3)}$. En la práctica clínica, los efectos adversos como la nefrotoxicidad y neurotoxicidad limitaron su uso por años; sin embargo, actualmente se emplea el colistimetato sódico como profármaco inactivo, el cual, en medio acuoso, se transforma en colistina disminuyendo así sus efectos nocivos ${ }^{(4)}$.

Se han identificado diferentes mecanismos de resistencia a esta polimixina; uno de ellos es el producto de modificaciones de los lipopolisacáridos de la membrana externa (en el lípido A) mediado por la adición de un 4-amino-4-desoxy-L-arabinosa (L-Ara4N) y la fosfoetanolamina (PEtN). Esta modificación del lípido A está regulada por los sistemas de dos componentes (TCS): PhoP-PhoQ (PhoPQ) y PmrA-PmrB (PmrAB), lo que da como resultado la reducción de la carga negativa de la membrana externa que genera resistencia a la colistina ${ }^{(1,5)}$.

De otra parte, la resistencia plasmídica a la colistina denominada mcr-1 (mobile colistin resistance), miembro de la familia de las enzimas fosfoetanolamina transferasas, capaz de modificar el sitio blanco de la colistina, disminuyendo su afinidad por el lípido $\mathrm{A}$ al adicionar fosfoetanolamina, fue descrita por primera vez en China el $2015^{(6)}$. Ese mismo año, en Vietnam, se reportó el primer caso clínico en una muestra diarreica, criopreservada hasta el 2008, en la cual se aisló una Shigella sonnei portadora del gen ${ }^{(7)}$. A la fecha, se han caracterizado 10 genes homólogos a mcr-1 y 21 variantes alélicas ${ }^{(8)}$. Este gen es transmitido por plásmidos, asociados principalmente al grupo de incompatibilidad X4 (IncX4), lo que permite su transferencia horizontal entre diversas especies de BGN ${ }^{(9)}$.

En el 2016, Arcilla et al., reportaron dos cepas de Escherichia coli productora de BLEE y portadoras del gen mcr-1, aislados de muestras diarreicas de viajeros holandeses provenientes del Perú, Colombia y Bolivia después de su

\section{MENSAJES CLAVE}

Motivación para realizar el estudio: Ante la posibilidad de subregistros de resistencia a la colistina en hospitales y clínicas del Perú, se utilizaron tres metodologías con alto rendimiento para determinar la frecuencia de resistencia a la colistina por mecanismos cromosómicos y plasmídicos.

Principales hallazgos: Se determinó el 7,2\% de aislados de $P$. aeruginosa resistentes a la colistina no portadores del gen mor-1.

Implicancias: Se determinó por primera vez la resistencia a la colistina en Pseudomona aeruginosa en el Perú.

retorno a Holanda ${ }^{(10)}$. En América Latina se ha reportado el gen $m c r-1$ en Argentina, Venezuela, Colombia, Ecuador y Brasil ${ }^{(11)}$. En el Perú, en el 2018, Ugarte et al., reportaron por primera vez aislados de E. coli y Klebsiella pneumoniae portadoras del $m c r-1{ }^{(12)}$. El mecanismo plasmídico mediado por el gen $m c r-1$, fue detectado por primera vez en $P$. aeruginosa y Acinetobacter baumannii en un estudio multicéntrico realizado en Pakistán el $2019^{(13)}$. En el Perú, hasta el momento, no se ha reportado resistencia a la colistina, tampoco la presencia del gen $\mathrm{mcr}-1$ en aislamientos de $P$. aeruginosa de origen clínico.

La emergencia de la resistencia a la colistina mediante la transferencia horizontal de plásmidos conjugativos genera una preocupación creciente en todo el mundo debido a su rápida diseminación. Con base en lo anterior, se realizó el presente estudio con el objetivo de determinar la frecuencia de la resistencia a la colistina por el método de elución de discos de colistina en caldo (CBDE, por sus siglas en inglés), la frecuencia de la expresión del gen $m c r-1$ por el método de difusión de discos combinados de colistina y EDTA (CDT, por sus siglas en inglés) y la presencia del gen $m c r-1$ por reacción en cadena de la polimerasa (PCR, por sus siglas en ingles), en aislados de $P$. aeruginosa, provenientes de tres establecimientos de salud en la ciudad de Lima, Perú.

\section{EL ESTUDIO}

Se realizó un estudio descriptivo transversal en el que se evaluaron aislados de $P$. aeruginosa de origen clínico, provenientes de tres establecimientos de salud de Lima (dos públicos y uno privado) desde enero del 2018 hasta octubre del 2019. Los aislados se encontraban criopreservados en el banco de cepas del Laboratorio de Resistencia a Antimicrobianos e Inmunopatología de los Laboratorios de Investigación y Desarrollo (LID) de la Universidad Peruana Cayetano Heredia (UPCH). 


\section{Detección fenotípica de la resistencia a la colistina}

Se recuperaron 97 aislados de $P$. aeruginosa en el medio selectivo Cetrimide (Liofilchem, Italia) e incubados por 24 horas a $35^{\circ} \mathrm{C}$. Se determinó la resistencia a la colistina mediante el método de CBDE, descrita por el Laboratorio de Referencia en Antimicrobianos de Argentina «Dr. Carlos G. Malbrán» ${ }^{(14)}$. Los aislados se clasificaron, en base a los resultados de la concentración mínima inhibitoria (CMI), intermedio (CMI $\leq 2 \mu \mathrm{g} / \mathrm{mL})$ o resistentes $(\mathrm{CMI} \geq 4 \mu \mathrm{g} / \mathrm{mL})$, para lo cual empleamos los puntos de corte establecidos en la guía M100-ED30: 2020 del Clinical and Laboratory Standard Institute (CLSI) ${ }^{(15)}$.

\section{Prueba de difusión de disco combinada}

Los aislamientos se inocularon sobre agar Müller Hinton (Merck, Alemania) a partir de un tubo con suero fisiológico a una concentración equivalente al tubo $n .^{\circ} 0,5$ de la escala de McFarland. Se emplearon dos discos de sensibilidad, uno con $10 \mu \mathrm{g}$ de colistina (Oxoid, Inglaterra) y el segundo con $10 \mu \mathrm{g}$ de colistina más $10 \mu \mathrm{L}$ de EDTA (Sigma-Aldrich, EUA) a una concentración de $100 \mathrm{mM}$. Una diferencia $\geq 3 \mathrm{~mm}$ entre los halos de inhibición de colistina más EDTA en comparación con la observada en el disco de colistina sola se consideró positivo para la expresión del gen $m c r-1$; el método fue estandarizado por Esposito et al ${ }^{(16)}$.

\section{Detección del gen $\boldsymbol{m c r}$-1}

Para la identificación molecular del gen, se extrajo el ADN genómico de los 97 aislados por el método de choque térmico y se realizó la PCR para el gen $m c r-1$ empleando los siguientes cebadores: forward: CLR5-F1 (5'-CGGTCAGTCCGTTTGTTC-3') y reverse: MCR-indel-R1 (5'-CTTGGTCGGTCTGTAGGG-3). La amplificación se realizó en las siguientes condiciones: desnaturalización inicial a $94^{\circ} \mathrm{C}$ por 5 minutos, seguido de 35 ciclos a $94^{\circ} \mathrm{C}$ por 30 segundos; $45^{\circ} \mathrm{C}$ por 30 segundos; $72{ }^{\circ} \mathrm{C}$ por 30 segundos y extensión final a $72{ }^{\circ} \mathrm{C}$ durante $10 \mathrm{~min}$, según la metodología descrita por Liu YY ${ }^{(6)}$. El tamaño del ADN se verificó con DNA ladder 100 bp (Thermo Scientific, EUA), considerando productos de $309 \mathrm{pb}$.

En todas las pruebas, para la validación de los resultados, se usó como control positivo una cepa de E. coli caracterizada molecularmente como portadora del gen $m c r-1$, perteneciente al Laboratorio de Resistencia a los Antimicrobianos e Inmunopatología y, como control negativo, se empleó una E. coli ATCC 25922.

\section{Análisis de datos}

Se utilizó el programa estadístico STATA, versión 16.0 (StataCorp, College Station, TX, EUA) para reportar frecuencias absolutas y el porcentaje de las variables de interés obtenidas del estudio.

\section{Aspectos éticos}

El protocolo se registró y aprobó en el Sistema Descentralizado de Información y Seguimiento a la Investigación (SIDISI:103444) - Dirección Universitaria de Investigación, Ciencia y Tecnología (DUICT); y, antes de su ejecución, el Comité de Ética de la UPCH (CIE-UPCH: CAREGORVEI-141-19) lo evaluó. El estudio siguió los lineamientos de las buenas prácticas y ética en investigación biomédica.

\section{HALLAZGOS}

Del total de 97 aislados de $P$. aeruginosa de origen clínico, el método de CBDE identificó 7 (7,2\%) aislados resistentes a la colistina con una CMI igual a $4 \mu \mathrm{g} / \mathrm{mL} ; 49$ (50,5\%) presentaron una CMI igual a $2 \mu \mathrm{g} / \mathrm{mL}$; y 41 aislados $(42,3 \%)$ presentaron una CMI menor o igual a $1 \mu \mathrm{g} / \mathrm{mL}$ y fueron catalogados como intermedio a la colistina. La concentración del antibiótico que inhibió al 50\% y 90\% de los aislados (CMI ${ }_{50}$ y CMI9o ) fue de $2 \mu \mathrm{g} / \mathrm{mL}$. Los aislamientos resistentes a la colistina se obtuvieron de diferentes muestras clínicas, como la secreción de heridas $(\mathrm{n}=2)$, sangre $(\mathrm{n}=3)$, orina $(\mathrm{n}=1)$ y heces $(n=1)$. Del total de los aislamientos resistentes a la colistina, 2 fueron del establecimiento de salud 1 (6,9\%, n = 29), 2 del establecimiento de salud $2(12,5 \%, \mathrm{n}=16)$ y 3 del establecimiento de salud $3(5,8 \%, \mathrm{n}=52)$ (Tabla 1$)$. El resultado del método CDT fue negativo para el total de cepas evaluadas. Asimismo, la prueba de PCR no detectó ningún aislamiento portador del gen $m c r-1$ (Tabla 2).

Tabla 1. Resistencia bacteriana a la colistina y características de los aislados clínicos.

\begin{tabular}{lccc}
\hline & Total & \multicolumn{2}{c}{ Colistina $^{\text {a }}$} \\
\cline { 3 - 4 } Característica & $(\mathbf{N}=\mathbf{9 7})$ & $\begin{array}{c}\text { Resistente } \\
(\mathbf{n}=7)\end{array}$ & $\begin{array}{c}\text { Intermedio } \\
(\mathbf{n}=\mathbf{9 0})\end{array}$ \\
\cline { 2 - 4 } & $\mathrm{N}(\%)$ & $\mathrm{n}(\%)$ & $\mathrm{n}(\%)$ \\
\hline Institución & & & \\
Centro de salud 1 & $29(29,9)$ & $2(6,9)$ & $27(93,1)$ \\
Centro de salud 2 & $16(16,5)$ & $2(12,5)$ & $14(87,5)$ \\
Centro de salud 3 & $52(53,6)$ & $3(5,8)$ & $49(94,2)$ \\
Tipo de muestra & & & \\
Aspirado bronquial & $4(4,1)$ & - & $4(100,0)$ \\
Bilis & $1(1,0)$ & - & $1(100,0)$ \\
Coprocultivo & $2(2,1)$ & $1(50,0)$ & $1(50,0)$ \\
Drenaje torácico & $1(1,0)$ & - & $1(100,0)$ \\
Esputo & $1(1,0)$ & - & $1(100,0)$ \\
Hemocultivo & $11(11,3)$ & $3(27,3)$ & $8(72,7)$ \\
Secreciones & $59(60,8)$ & $2(3,4)$ & $57(96,6)$ \\
Urocultivo & $18(18,6)$ & $1(5,6)$ & $17(94,4)$ \\
\hline
\end{tabular}

a Método de elución de discos de colistina en caldo (CBDE) 
Tabla 2. Método de elución de discos de colistina en caldo, prueba de difusión de disco combinada y reacción en cadena de la polimerasa para mcr-1 en Pseudomonas aeruginosa fenotipo colistina resistente.

\begin{tabular}{|c|c|c|c|c|c|}
\hline Código de cepa & Centro de salud & Tipo de muestra & CBDE & CDT & PCR gen $m c r-1$ \\
\hline 2041 & 1 & Cultivo de secreción & $4 \mathrm{mg} / \mathrm{mL}(\mathrm{R})$ & $0 \mathrm{~mm}$ & Negativo \\
\hline 2043 & 1 & Urocultivo & $4 \mathrm{mg} / \mathrm{mL}(\mathrm{R})$ & $0 \mathrm{~mm}$ & Negativo \\
\hline 1002 & 2 & Coprocultivo & $4 \mathrm{mg} / \mathrm{mL}(\mathrm{R})$ & $0 \mathrm{~mm}$ & Negativo \\
\hline 1005 & 2 & Hemocultivo & $4 \mathrm{mg} / \mathrm{mL}(\mathrm{R})$ & $2 \mathrm{~mm}$ & Negativo \\
\hline 430 & 3 & Cultivo de secreción & $4 \mathrm{mg} / \mathrm{mL}(\mathrm{R})$ & $1 \mathrm{~mm}$ & Negativo \\
\hline 631 & 3 & Hemocultivo & $4 \mathrm{mg} / \mathrm{mL}(\mathrm{R})$ & $1 \mathrm{~mm}$ & Negativo \\
\hline 806 & 3 & Hemocultivo & $4 \mathrm{mg} / \mathrm{mL}(\mathrm{R})$ & $1 \mathrm{~mm}$ & Negativo \\
\hline
\end{tabular}

CBDE: Método de elución de discos de colistina en caldo; CDT: prueba de difusión de disco combinada; PCR: reacción en cadena de la polimerasa; R: resistente

\section{DISCUSIÓN}

Los resultados del presente estudio muestran que el 7,2\% de los aislados de $P$. aeruginosa fueron resistentes a la colistina. Este hallazgo es similar al 5,2\% reportado para América Latina ${ }^{(17)}$ y Europa $4 \%{ }^{(3)}$. En el Perú, no se ha reportado resistencia a la colistina en $P$. aeruginosa, antes de este estudio ${ }^{(18)}$.

Diversos estudios han determinado que los métodos convencionales empleados en los antibiogramas, como difusión de discos en agar, Epsilon test, incluso los sistemas comerciales como el VITEK (Biomerieux), Phoenix (Becton Dickinson), empleados en los hospitales en todo el país, generan falsa susceptibilidad a la colistina, lo que podría estar conduciendo a subregistros de la resistencia real ${ }^{(19)}$.

Dada la importancia actual del antibiótico y los problemas técnicos relacionados con la determinación de resistencia, ameritaba un estudio con métodos de probada efectividad. La prueba de CBDE es una buena alternativa en términos de rentabilidad, eficacia y desempeño, y puede aplicarse en la rutina del laboratorio de microbiología clínica ${ }^{(3,19)}$. El estudio realizado por Humphries, et al. avala la aplicación de esta prueba en $P$. aeruginosa con una concordancia esencial del $94,4 \%$ y una concordancia categórica del $97,9 \%$ con las CMI obtenidas por el método de microdilución en caldo, considerada como la prueba de referencia. Con base en estos resultados, el Subcomité de Pruebas de Susceptibilidad Antimicrobiana (AST) del CLSI aprobó el método de CBDE para las pruebas de resistencia en enterobacterias y $P$. aeruginos $a^{(18,20)}$.

El empleo de quelantes como el EDTA para la inhibición de la fosfoetanolamina transferasa producida por el gen $m c r-1$ se ha difundido en los laboratorios de microbiología clínica. La prueba de CDT para la detección presuntiva de la expresión fenotípica de las variantes del gen $m c r$ mediante alteración del potencial zeta mostró resultados prometedores, pues constituye una alterativa rápida y económica en aislados de $E$. coli ${ }^{(16)}$. Nuestros resultados mostraron que los aislados de $P$. aeruginosa con un fenotipo resistente a la colistina $(\mathrm{CMI} \geq 4 \mu \mathrm{g} / \mathrm{mL})$ no tuvieron una diferencia $\geq 3 \mathrm{~mm}$ en el diámetro de la zona de inhibición del disco de colistina/EDTA en comparación con el disco de colistina, característica que se relaciona con la ausencia del gen $m c r-1$. Estos resultados son similares a los publicados por Abd El-Baky, et al., quienes encontraron que el 50,0\% de $P$. aeruginosa con una $\mathrm{CMI} \geq 32 \mu \mathrm{g} / \mathrm{mL}$ y $\mathrm{CDT}$ negativo no presentaban el gen $m c r-1^{(5)}$.

En el presente estudio, el cribado del gen $m r c-1$ mediante PCR fue negativo para el total de cepas evaluadas, ello indica que la resistencia a la colistina que hemos detectado podría deberse a la presencia de variantes alélicas del gen o a mecanismos cromosómicos que no hemos podido detectar con la metodología empleada. Es importante considerar que la identificación molecular del gen $m c r-1$ y sus variantes no necesariamente confirman la resistencia a la colistina en las bacterias que lo portan, ya que pueden presentarse fenotipos susceptibles $^{(8)}$.

Recientemente, se identificó que el principal mecanismo responsable de la resistencia cromosómica a la colistina en BGN es el resultado de mutaciones específicas, estímulos ambientales e inactivación por inserción en el sistema de dos componentes (TCS) PhoP-PhoQ (PhoPQ) y PmrA-PmrB $(\mathrm{PmrAB})$, que conducen a una sobreexpresión del operón pmrHFIJKLM. Esto trae como consecuencia la adición de L-Ara4Ns y PEtNs en el lípido A de la membrana externa de P. aeruginosa, la cual disminuye la carga neta negativa de los residuos de fosfato que afecta la afinidad de la colistina ${ }^{(4,5)}$.

A pesar de la preocupante emergencia de variantes de P. aeruginosa resistentes a la colistina, la implementación de pruebas de susceptibilidad sensibles y específicas en el trabajo rutinario de los laboratorios de microbiología clínica ha sido lenta. En este estudio utilizamos protocolos bien definidos que combinan pruebas de susceptibilidad fenotípica y métodos moleculares. Sin embargo, existen limitaciones que consideramos conveniente resaltar; los aislamientos incluidos en el estudio se obtuvieron de tres establecimientos de salud durante un periodo de tiempo específico, por lo tanto, nuestros resultados no pueden inferirse a la situación actual del ámbito hospitalario; asimismo, no se evaluaron los genes homólogos a $m c r-1$; tampoco se caracterizaron los mecanismos cromosómicos implicados en la resistencia a la colistina. 
En conclusión, con el método de CBDE se identificó 7,2\% de aislados clínicos de $P$. aeruginosa resistentes a la colistina, este hecho constituye el primer reporte de resistencia a este antimicrobiano en el Perú. El hallazgo muestra la necesidad de implementar, en nuestro país, la vigilancia de la resistencia a la colistina en $P$. aeruginosa mediante la aplicación de metodologías adecuadas.

\section{REFERENCIAS BIBLIOGRÁFICAS}

1. Lee JY, Park YK, Chung ES, Na IY, Ko KS. Evolved resistance to colistin and its loss due to genetic reversion in Pseudomonas aeruginosa. Sci Rep. 2016; 6: 25543. doi: 10.1038/srep25543.

2. Tacconelli E, Carrara E, Savoldi A, Harbarth S, Mendelson M, Monnet DL, et al. Discovery, research, and development of new antibiotics: the WHO priority list of antibiotic-resistant bacteria and tuberculosis. Lancet Infect Dis. 2018;18(3):318-27. doi: 10.1016/S1473-3099(17)30753-3.

3. Li Z, Cao Y, Yi L, Liu JH, Yang Q. Emergent Polymyxin Resistance: End of an Era?. Open Forum Infect Dis. 2019;6(10):ofz368. doi:10.1093/ ofid/ofz368.

4. Grégoire N, Aranzana-Climent V, Magréault S, Marchand S, Couet W. Clinical Pharmacokinetics and Pharmacodynamics of Colistin. Clin Pharmacokinet. 2017;56(12):1441-60. doi: 10.1007/s40262-017-0561-1.

5. Abd El-Baky RM, Masoud SM, Mohamed DS, Waly NG, Shafik EA, Mohareb DA, et al. Prevalence and Some Possible Mechanisms of Colistin Resistance Among Multidrug-Resistant and Extensively Drug-Resistant Pseudomonas aeruginosa. Infect Drug Resist. 2020;13:323-332. doi:10.2147/IDR.S238811.

6. Liu YY, Wang Y, Walsh TR, Yi LX, Zhang R, Spencer J, et al. Emergence of plasmid-mediated colistin resistance mechanism MCR-1 in animals and human beings in China: a microbiological and molecular biological study. Lancet Infect Dis. 2016;16(2):161-8. doi: 10.1016/S1473-3099(15)00424-7.

7. Pham-Thanh D, Thanh-Tuyen H, Nguyen-Thi-Nguyen T, Chung-The H, Wick RR, Thwaites GE, et al. Inducible colistin resistance via a disrupted plasmid-borne mcr-1 gene in a 2008 Vietnamese Shigella sonnei isolate. J Antimicrob Chemother. 2016;71(8):2314-2317. doi:10.1093/jac/dkw173.

8. Wang C, Feng Y, Liu L, Wei L, Kang M, Zong Z. Identification of novel mobile colistin resistance gene mcr-10. Emerg Microbes Infect. 2020;9(1):508-516. doi:10.1080/22221751.2020.1732231.

9. Gröndahl-Yli-Hannuksela K, Lönnqvist E, Kallonen T, Lindholm L, Jalava J, Rantakokko-Jalava K, et al. The first human report of mobile colistin resistance gene, mcr-1, in Finland. APMIS. 2018;126(5):413-417. doi: 10.1111/apm.12834.

10. Arcilla MS, Hattem JMvan, Matamoros S, Melles DC, Penders J, Jong MD de, et al. Dissemination of the mcr-1 colistin resistance gene. Lancet Infect Dis. 2016;16(2):147-9. doi:10.1016/S1473-3099(15)00541-1.

11. Quiroga C, Nastro M, Di Conza J. Current scenario of plasmid-mediated colistin resistance in Latin America. Rev Argent Microbiol. 2019;51(1):93100. doi: 10.1016/j.ram.2018.05.001.
Contribuciones de autoría: Todos los autores han participado en la idea de la investigación, concepción del artículo; recolección de datos y material de estudio; y redacción del artículo. Todos los autores aprobaron la versión final del manuscrito.

Financiamiento: El estudio fue financiado con recursos del Laboratorio de Resistencia a Antimicrobianos-LID-UPCH.

Conflictos de interés: Los autores no tienen ningún conflicto de interés que declarar.

12. Ugarte Silva RG, Olivo López JM, Corso A, Pasteran F, Albornoz E Blácido ZPS. Resistencia a colistín mediado por el gen mcr-1 identificado en cepas de Escherichia coli y Klebsiella pneumoniae. Primeros reportes en el Perú. An Fac Med. 2018;79(3):213-7. doi: 10.15381/ anales.v79i3.15313.

13. Hameed F, Khan MA, Muhammad H, Sarwar T, Bilal H, Rehman TU, et al. Plasmid-mediated mcr-1 gene in Acinetobacter baumannii and Pseudomonas aeruginosa: first report from Pakistan. Rev Soc Bras Med Trop. 2019;52:e20190237. doi: 10.1590/0037-8682-0237-2019.

14. Servicio Antimicrobianos - INEI-MALBRAN. Metodo de elusion de discos de colistin. Buenos Aires; 2017. Disponible en: http://antimicrobianos.com.ar/ATB/wp-content/uploads/2017/09/Protocolo-Met-deEluci\%C3\%B3n-de-Discos-de-COL-version3-Nov2017.pdf.

15. Clinical and Laboratory Standards Institute. 2020. Performance standards for antimicrobial susceptibility testing; 30th informational supplement. Clinical and Laboratory Standards Institute, Wayne, PA. 2020.

16. Esposito F, Fernandes MR, Lopes R, Muñoz M, Sabino CP, Cunha MP, et al. Detection of Colistin-Resistant MCR-1-Positive Escherichia coli Using Inhibition by EDTA and Zeta Potential Assays. J Clin Microbiol. 2017;55(12):3454-3465. doi:10.1128/JCM.00835-17.

17. Karlowsky JA, Kazmierczak KM, Bouchillon SK, Jonge BLM de, Stone GG, Sahm DF. In Vitro Activity of Ceftazidime-Avibactam against Clinical Isolates of Enterobacteriaceae and Pseudomonas aeruginosa Collected in Latin American Countries: Results from the INFORM Global Surveillance Program, 2012 to 2015. Antimicrob Agents Chemother. 2019;63(4). doi:10.1128/AAC.01814-18.

18. Fernández DF, García C, Zegarra J, Granados L. Susceptibilidad antimicrobiana en aislamientos de secreción endotraqueal en la unidad de cuidados intensivos de un hospital nacional de Lima, 2016. Rev Medica Hered. 2017;28(4):236-236. doi: 10.20453/rmh.v28i4.3223.

19. Sekyere JO. Mcr colistin resistance gene: a systematic review of current diagnostics and detection methods. MicrobiologyOpen . 2019;8(4):e00682. doi: 10.1002/mbo3.682.

20. Humphries RM, Green DA, Schuetz AN, Bergman Y, Lewis S, Yee R, et al. Multicenter Evaluation of Colistin Broth Disk Elution and Colistin Agar Test: a Report from the Clinical and Laboratory Standards Institute. J Clin Microbiol. 2019;57:e01269-19. doi: 10.1128/JCM.01269-19. 